\title{
Cortical influences drive amyotrophic lateral sclerosis
}

\author{
Andrew Eisen, ${ }^{1}$ Heiko Braak, ${ }^{2}$ Kelly Del Tredici, ${ }^{2}$ Roger Lemon, ${ }^{3}$ Albert C Ludolph, ${ }^{4}$ \\ Matthew C Kiernan ${ }^{5}$
}

'Division of Neurology, University of British Columbia, Vancouver, British Columbia, Canada

${ }^{2}$ Clinical Neuroanatomy Section, Department of Neurology,

Center for Biomedical Research, University of Ulm, Ulm, Baden-

Württemberg, Germany

${ }^{3}$ Sobell Department of Motor Neuroscience and Movement Disorders, Sobell Department of Motor Neuroscience and Movement Disorders, UCL Institute of Neurology, Queen

Square, London, UK

${ }^{4}$ Department of Neurology, University of Ulm, Ulm, Germany ${ }^{5}$ Brain and Mind Centre, The University of Sydney, Sydney, NSW, Australia

\section{Correspondence to}

Dr Andrew Eisen, Division of Neurology, University of British Columbia, Vancouver, British Columbia, Canada; eisen@mail. ubc.ca

Received 15 March 2017 Revised 26 April 2017 Accepted 2 May 2017

\section{(1) corsmank}

To cite: Eisen A, Braak H, Del Tredici K, et al. J Neurol Neurosurg Psychiatry 2017:88:917-924.

\section{ABSTRACT}

The early motor manifestations of sporadic amyotrophic lateral sclerosis (ALS), while rarely documented, reflect failure of adaptive complex motor skills. The development of these skills correlates with progressive evolution of a direct corticomotoneuronal system that is unique to primates and markedly enhanced in humans. The failure of this system in ALS may translate into the split hand presentation, gait disturbance, split leg syndrome and bulbar symptomatology related to vocalisation and breathing, and possibly diffuse fasciculation, characteristic of ALS. Clinical neurophysiology of the brain employing transcranial magnetic stimulation has convincingly demonstrated a presymptomatic reduction or absence of short interval intracortical inhibition, accompanied by increased intracortical facilitation, indicating cortical hyperexcitability. The hallmark of the TDP-43 pathological signature of sporadic ALS is restricted to cortical areas as well as to subcortical nuclei that are under the direct control of corticofugal projections. This provides anatomical support that the origins of the TDP-43 pathology reside in the cerebral cortex itself, secondarily in corticofugal fibres and the subcortical targets with which they make monosynaptic connections. The latter feature explains the multisystem degeneration that characterises ALS. Consideration of ALS as a primary neurodegenerative disorder of the human brain may incorporate concepts of prion-like spread at synaptic terminals of corticofugal axons. Further, such a concept could explain the recognised widespread imaging abnormalities of the ALS neocortex and the accepted relationship between ALS and frontotemporal dementia.

\section{INTRODUCTION}

Amyotrophic lateral sclerosis (ALS) includes sporadic presentations (95\% of all cases), recognised to be associated with a growing number of pathogenic mutations in addition to a heterogeneous hereditary group (5\% of all cases) most of which clinically resemble the sporadic disease but display diverse pathologies. ${ }^{1}$ Patients with SOD1 mutations have predominantly SOD1-proteinopathy and those with FUS mutations have a FUS-proteinopathy. But the hallmark of sporadic ALS, with which we are primarily concerned with in this review, is abnormality in the predominantly intranuclear transactive response DNA-binding protein 43 (TDP-43).

This protein undergoes nuclear clearance followed by defective phosphorylation, conformational change and ubiquitination, which ultimately prevents re-entry into the nucleus. ${ }^{2}$ The gradual but complete intranuclear loss of TDP-43 within select nerve cells prevents the protein from fulfilling its physiological functions ('loss of function'). ${ }^{3}$ By contrast, abnormal cytoplasmic TDP-43 aggregates fail to undergo cellular clearance, and it is presumed that for a limited time a noxious soluble form of the protein exists in the cytoplasm that is also capable of entering distal portions of axons ('gain of function'), thereby affecting bulbar and spinal motoneurons. ${ }^{4}$

The mechanisms surrounding the earliest pathogenic events within involved neurons remain unknown. But it is neurons with corticofugal projections and their recipients receiving a direct, monosynaptic, neocortical synaptic input that predominantly develop TDP-43 pathology, while subcortical neurons with corticopetal connectivities are virtually spared. ${ }^{5}$ The corticofugal primacy in TDP-43 pathology supports Charcot, who was the first to postulate a cortical origin of ALS. ${ }^{6}$ Years later, quantitative pathological studies to test Charcot's hypothesis, by measuring the cross-sectional areas of pyramidal cells in layer $\mathrm{V}$ of the foot and tongue precentral areas, showed no correlation between them and numbers of surviving hypoglossal and spinal cord motoneurons. It was concluded that functionally related cortical and lower motoneurons might degenerate independently. ${ }^{7}$

The present review will contend that ALS is a human disorder primarily affecting the brain and predominantly mediated via the neocortex..$^{8-11}$ Evidence will be presented to consider (1) cogent aspects of descending human motor pathways, in particular the human corticomotoneuronal (CM) system; (2) clinical ALS correlates that are best explained on the basis of cortical primacy; (3) support from clinical neurophysiology; (4) support derived from TDP-43 neuropathology and (5) imaging studies supportive of ALS as a primary cortical and secondary subcortical disease. If this view is correct, there are implications for future research and therapeutic avenues.

\section{The human CM system}

Key to consideration of primacy of the cerebral cortex in ALS is the development of a concept of the CM system. Direct connections between specific cortical projection cells and their bulbar and spinal targets, the $\alpha$-motoneurons, were discovered $>60$ years ago. They form a component of the corticobulbar tract (CBT) and corticospinal tract (CST) and have multiple functions sharing one characteristic, namely direct control of bulbar and spinal cord activity through monosynaptic CM connections 
in cooperation with other indirectly synapsing neurons. ${ }^{12-14}$ The CM system subserves special functions, including adaptive complex motor behaviours, largely limited to non-human primates and humans. ${ }^{14-17}$ It has been repeatedly suggested that the increased influence of human corticospinal and corticobulbar systems has paralleled a decrease in the importance of the rubrospinal tract, a descending pathway that is generally well developed in many non-human models. ${ }^{18}$

Most CBT/CST axons originate from projection neurons of layer $\mathrm{V}$ of the primary motor and sensory neocortex. A smaller proportion arises from premotor regions, the supplementary motor cortex and somatosensory association fields. The numeric relationship between axons of CM cells and $\alpha$-motoneurons is not one-to-one. Each $\alpha$-motoneuron receives input from many $\mathrm{CM}$ cells (convergence) and a single CM cell innervates many different $\alpha$-motoneurons belonging to different synergistic muscle groups (divergence). ${ }^{14}$ In humans, fast-conducting axons comprise about $8 \%$ of the axons of the pyramidal tract, and these will include many with CM connections. CM cells synapse with all motoneuron pools except those innervating the external ocular muscles and sphincter muscles which coincidentally remain relatively spared in ALS. ${ }^{18-20}$

Skilled movements involving different combinations of muscles involve separate populations of CM cells, which are widely distributed within the primary motor cortex. ${ }^{1621} \mathrm{CM}$ synapses in humans probably contribute different proportions of excitatory input to different motoneuron pools, but may contribute a decisive proportion of the synaptic input to muscles involved in thumb opposability and skilled hand function, with different CM populations subserving precision versus power grip. ${ }^{1622}$ In gait, there is evidence that the corticospinal input may be particularly important for locomotion over uneven or challenging terrains and activities requiring pedal agility. ${ }^{23}$ The same conclusion may apply to complex vocalisation and breathing, and other multineuronal bulbar functioning. ${ }^{24}$ Disruption of these complex functions, in turn, is uniquely reflected in the ALS, as the split hand syndrome, ${ }^{25}$ the split leg

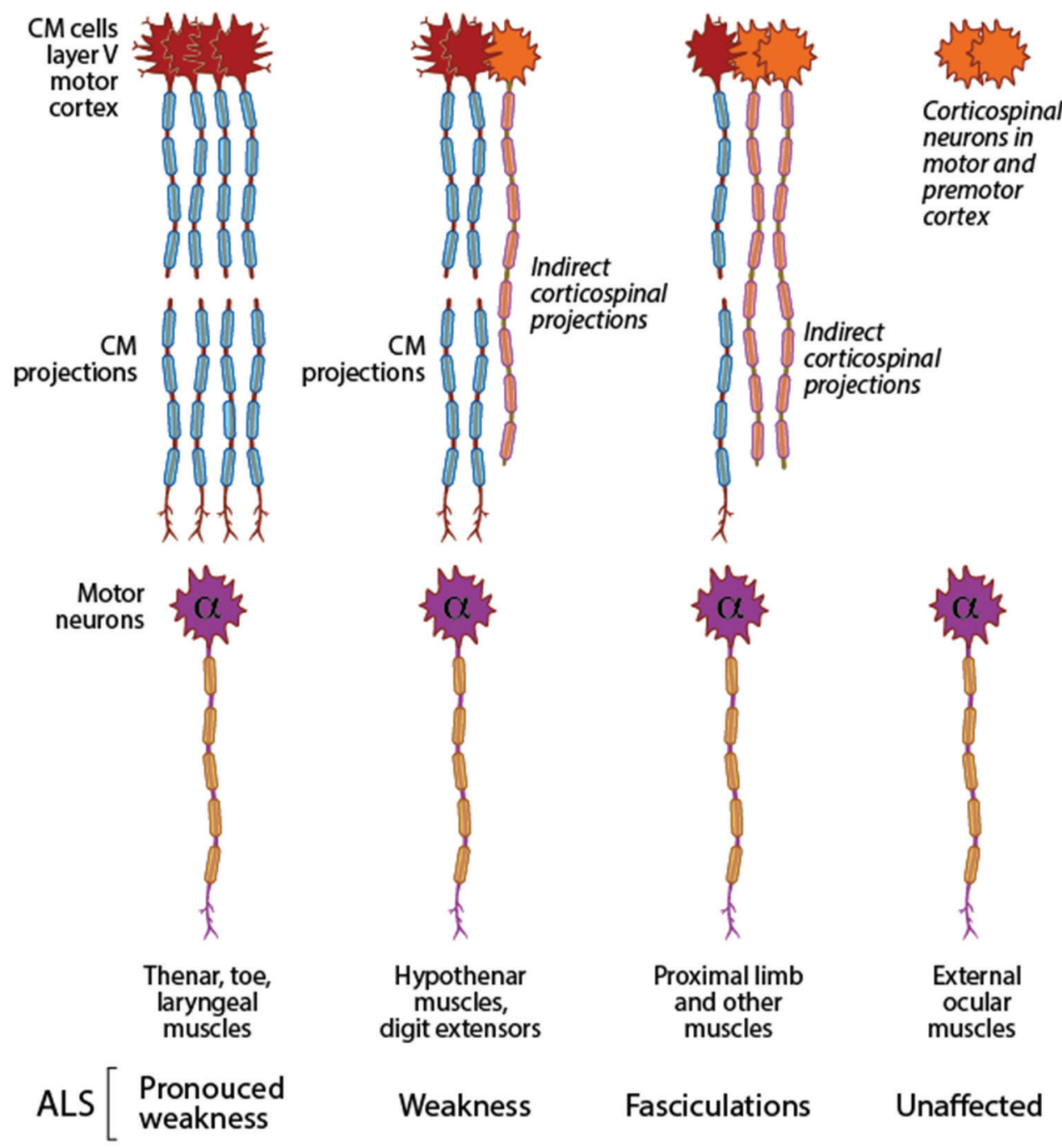

Figure 1 Corticomotoneuronal (CM) cells synapse with all motoneuron pools except those innervating the external ocular muscles and sphincter muscles which coincidentally remain relatively spared in amyotrophic lateral sclerosis (ALS). CM synapses in humans likely may contribute a decisive proportion of the synaptic input to muscles involved in skilled hand function, pedal agility, complex vocalisation and breathing, and other multineuronal bulbar functioning. CM input is strongest to the low-threshold, early recruited motor units, the same ones in which ALS fasciculations can be initially recorded, and are thus appreciated early in a wider group of muscles than those mediating skilled motor activity. 
syndrome ${ }^{26}$ and possibly specific combinations of bulbar manifestations ${ }^{17}$ (figure 1).

\section{Clinical syndromes that point to involvement of the cerebral cortex}

Prior to developing overt clinical manifestations in terms of muscle wasting or weakness, if asked, patients with ALS are frequently aware of a variety of subtle problems. ${ }^{17} 27$ However, to date this has not been systematically studied. Examples include impairment of fine fractionated hand control and orofacial movements, such as those performed during object manipulation and phono-articulation, and impaired skilled walking, as is required to navigate uneven territory. These attributes are primarily subserved by cortical motor networks involving in particular the prefrontal areas, the premotor and primary motor neocortex, posterior parietal cortex and their CBT/CST derivatives. These are all prime pathological targets of the disease, which disrupt these heavily interconnected cortical motor networks, with resulting syndromic deficits such as the split hand syndrome, gait dysfunction and other complex clinical dysfunctions involving vocalisation and breathing that are less well understood, but which too can be related to involvement of the cerebral cortex.

\section{Split hand syndrome}

The complexity of CM control, driven by evolutionary demands, parallels advances in dexterity across primate species. ${ }^{14}$ In humans, the marked vulnerability of the hand-CM system is manifest most obviously in the 'split hand' syndrome. The muscles involved underlie pincer and precision grips (prehensility), which are dependent on CM integrity. ${ }^{141618}$ Early hand dysfunction preferentially affects the 'thenar hand', including the abductor pollicis brevis and first dorsal interosseous muscles (figure 1). In contrast, there is relative sparing of the hypothenar muscles (abductor digiti minimi). This dissociated atrophy of the intrinsic hand muscles is virtually unique to the ALS. The marked differences in thenar versus hypothenar weakness are not fully attributable to peripheral nerve pathology.

\section{Gait}

A clumsy, hesitant gait is a common early feature, often manifested as a subtle partial foot drop (spinal motoneuron foot weakness that can be compensated). Neurophysiological and imaging studies have provided evidence that the motor neocortex plays a significant role in human gait, even during undemanding steady-state walking. ${ }^{23}$ The predominance of ankle dorsiflexion weakness compared with plantar flexion is commonly ascribed to the effect of gravity. However, a better explanation lies in the much greater CM drive to ankle dorsiflexors compared with ankle plantar flexors (split leg syndrome), which are more under the control of subcortical structures.

\section{Bulbar syndromes}

Bulbar ALS syndromes due to cortical primacy are more difficult to identify given that by the time deficits become obvious there is a significant involvement of $\alpha$-motoneurons. This may reflect the relatively short corticobulbar CM connections compared with corticospinal CM connections. Human vocalisation is free-flowing with intricately varied pitch and speed, requiring fine motor control of the entire respiratory motor complex. ${ }^{28}$ There are direct CM projections from motor cortex (area 4) to the nucleus ambiguus in humans ${ }^{19} 20$ and stimulation of this area elicits large, short-latency responses in laryngeal muscles. ${ }^{29}{ }^{30}$ Conclusive proof that these result from monosynaptic CM connections is difficult to demonstrate. Direct projections to laryngeal motoneurons from either area 4 or area 6 are lacking in the non-human primate. ${ }^{30}$ In humans, there is also evidence for direct corticospinal influences over respiratory muscles. ${ }^{28}$ Bulbar-onset cases most obviously impair vocalisation (dysarthria) and swallowing (dysphagia), but there is probably also significant impact of respiratory control as it relates to vocalisation.

\section{Frontotemporal dementia}

The association of frontotemporal dementia (FTD) and ALS clearly reflects primary cortical pathology. Interestingly, Charcot suggested that dementia was never associated with ALS, ${ }^{31}$ and historically a perception remains, even among neurologists, that cognitive deficits are not associated with the disease. ${ }^{32}$ However, genetic and neuropathological studies, combined with sophisticated imaging and neuropsychology, confirm frontotemporal dysfunction in a significant number of patients, with $10-15 \%$ of cases demonstrating overt dementia. ${ }^{33}$ In advanced disease, pathological changes within susceptible nerve cells of the cerebral cortex develop in anteromedial portions of the temporal lobe (entorhinal region and hippocampal formation), the integrity of which is crucial for effective learning and intact memory functioning. ${ }^{5}$

Separately, there is a subset of patients with ALS who do not manifest FTD, but in whom social cognition, particularly 'theory of mind', may be affected. ${ }^{34}$ This impairment may be related to dysfunctions of the neocortical mirror neuron system and other cortical regions related to action processing and understanding. ${ }^{35}$

\section{Fasciculation}

Fasciculations are not specific to ALS, but unlike other diseases, they are typically diffuse and profuse, and may precede the development of muscle weakness and wasting. Indeed, the absence of clinical or electrophysiologically recorded fasciculation makes the diagnosis of ALS questionable. ${ }^{36}$

ALS fasciculation probably has several different neural generators located from the motor cortex to the distal nerve terminal. However, data derived from transcranial magnetic stimulation (TMS) suggest that the CM input is probably strongest to the low-threshold, early recruited motor units, which are the same ones in which ALS fasciculations can be initially recorded. ${ }^{37}$ Thus, although relatively few spinal motoneuron pools receive a large enough CM input to result in significant weakness early in ALS, fasciculations in low-threshold motor units might be expected to appear across a much wider group of muscles, as is frequently seen early in ALS (figure 1).

\section{Oculomotor deficits}

Since it is generally established that oculomotor motoneurons do not receive direct projections from the cortex, ${ }^{19} 20$ if ALS results from pathological processes involving such direct projections, then it is important to determine whether there is oculomotor weakness in patients with ALS. The clinical and neurophysiological findings demonstrate that while there are deficits, these reflect problems with higher-level control and are not indicative of the motoneuron loss or weakness that characterises ALS in, for example, the hand and the foot. Oculomotor abnormalities occur in a two-phase manner. ${ }^{38}$ In the first phase, deficits affecting executive ('frontal') control of eye movements develop, that is, increased error rates in delayed saccade and antisaccade tasks, a reduction of rapidly voluntary gaze shifts and an 
increased incidence of saccadic intrusions occur. These ocular dysfunctions have been attributed to cell loss in the agranular motor neocortex, including the supplementary eye fields in Brodmann area $6^{39}$; but, because oculomotor nuclei do not receive direct projections from the cortex, it is difficult to be certain that this pathology is attributable to loss of corticofugal projections. Furthermore, as noted below, these nuclei do not display TDP-43 inclusions. ${ }^{40}$

The same frontal deficits also occur later in the disease, with the addition of other pathological features similar to those observed with pathology of the precerebellar nuclei, ${ }^{38}$ and are due to perturbation of brainstem circuits participating in control of eye movements (reticular formation, precerebellar nuclei). ${ }^{38}$

\section{NEUROPHYSIOLOGICAL CONSIDERATIONS}

In terms of objective changes in cortical function in the disease, paired-pulse TMS has provided key insights. Specifically, paired pulse TMS studies have identified reduction or absence of short interval intracortical inhibition (SICI) in patients, accompanied by increased intracortical facilitation. ${ }^{41}$ Taken together, these changes indicate the development of cortical hyperexcitability. In terms of other contributing influences, involvement of transcallosal pathways has been identified, ${ }^{42}$ which is thought to underlie the development of mirror movements. ${ }^{34}$

In terms of primacy of the cortical versus bulbar and spinal involvement, features of cortical hyperexcitability, heralded by reduction in SICI, have been identified during the early phases of ALS, ${ }^{43}$ preceding the clinical onset of bulbar/spinal motor dysfunction by approximately 3-6 months. ${ }^{44}$ Abnormalities of SICI and intracortical facilitation have also been confirmed in atypical ALS phenotypes, including the pure bulbar/spinal motor presentations of flail arm and flail leg variants, while at the other end of the disease spectrum, SICI abnormalities become evident in primary lateral sclerosis. The possibility that changes in SICI may develop as compensation in response to bulbar/spinal motoneuron degeneration and loss has essentially been excluded through studies undertaken in patients diagnosed with ALS mimics, such as spinobulbar muscular atrophy, where SICI changes were not evident, despite a comparable peripheral disease burden. Separately, temporary pseudonormalisation of SICI with riluzole, an anti-glutaminergic treatment licensed in ALS, provided further support for a pathogenic role of cortical hyperexcitability. ${ }^{45}$

Further neurophysiological support for the intrinsic role of cortical dysfunction has been derived from earlier single-pulse TMS approaches. Longitudinal studies in ALS established initial reductions of motor thresholds, followed in turn by the eventual development of cortical inexcitability in later phases of the disease, corresponding with possible loss of synaptic connections made by CBT/CST axons. Increase in amplitude of motor-evoked potentials, as well as significant reduction in the duration of the cortical silent period, has also been reported as early changes, again consistent with cortical hyperexcitability. Similar to earlier discussions, a reduction of the cortical silent period duration appears to be a specific feature of ALS, remaining normal in mimic disorders such as spinobulbar muscular atrophy, acquired neuromyotonia and distal hereditary motor neuronopathy with pyramidal features. ${ }^{46}$ The precise mechanism underlying this reduction in the cortical silent period remains to be established. Changes in ipsilateral cortical silent period have similarly been identified in ALS, evident in patients lacking signs of cortical involvement. ${ }^{47}$ These changes seem likely to be driven through involvement of transcallosal glutamatergic fibres projecting onto inhibitory interneurons in the non-stimulated motor cortex and appear to represent contributing factors to the cascade linked to the pathogenesis of the disease. ${ }^{42}$

These changes in neurophysiology revealed by TMS have more recently been identified in FTD, although less marked and thereby providing further support for its overlapping continuum of it with ALS. ${ }^{48}$ Widespread accumulation of TDP-43-positive inclusions in cortical neurons, a neuropathological hallmark of both FTD and sporadic ALS, supports the links between cortical dysfunction and ALS pathogenesis. In terms of understanding this process, it appears to be possible that cortical hyperexcitability may induce the spinal motoneuron degeneration via a glutamate-mediated excitotoxic mechanism. ${ }^{49}$ A number of molecular properties render $\alpha$-motoneurons prone to glutamate toxicity. Specifically, increased expression of the $\mathrm{Ca} 2+$ permeable glutamate receptor, $\alpha$-amino-3-hydroxy-5-methyl-4-isoxazoleproprionic acid, has been reported in ALS $\alpha$-motoneurons, with aberrant activity of the inositol 1,4,5-triphosphate receptor 2 gene, resulting in excessive $\mathrm{Ca} 2+$ accumulation following glutamate stimulation that potentially may explain the increased sensitivity of $\alpha$-motoneurons to glutamate excitotoxicity. ${ }^{5051}$

\section{Neuropathological considerations}

The hallmark abnormal nuclear clearance and the development of cytoplasmic TDP-43-positive inclusions in ALS are confined to specific projection neurons, characterised by axons that are disproportionately long, compared with the dimensions of their soma. In the primary motor cortex, pyramidal cells in layer $\mathrm{Vb}$, including the giant cells of Betz, are especially susceptible and are involved early in the disease. ${ }^{4052}$ This is also true of the large $\alpha$-motoneurons of the lower brainstem and spinal cord. ${ }^{4} 40$ Moreover, non-motor cells such as the projection neurons of the striatum, of the parvocellular red nucleus and of the inferior olive are also vulnerable.

By contrast, many neuronal types appear remarkably resistant to the development of cytoplasmic TDP-43-positive inclusions. These include motoneurons of the dorsal glossopharyngeal and vagal nuclei as well as projection cells of the parasympathetic and sympathetic nuclei in the spinal cord, which seldom or never develop TPD-43-positive lesions. ${ }^{40}$ Similarly, motor nuclei controlling extrinsic eye muscles, which are only indirectly influenced by the cerebral cortex and receive their contacts essentially from non-cortical fibres of the medial longitudinal fascicle, ${ }^{53}$ are spared until late in the disease course and remain nearly intact. In contrast, the motor nuclei of cranial nerves V, VII and XI-XII develop TDP-43 inclusions. ${ }^{8} 40$

\section{Spreading of TDP-43 pathology and neuropathological staging}

Neuropathological staging of ALS derived from TDP-43-immunopositive pathology has suggested that characteristic regional distribution patterns exist and develop in a time-dependent manner related to disease progression (figure 2). ${ }^{48}$ In stage 1 , the earliest abnormalities are in Betz pyramidal cells and in $\alpha$-motoneurons of the lower brainstem and spinal cord (motor dysfunction). ${ }^{40}$ During stage 2 , lesions begin to appear in cortical premotor areas and adjoining prefrontal fields, in parvocellular portions of the red nucleus, and in the inferior olivary nucleus (impairment of cerebellar function). In stage 3, the pathology develops further in the prefrontal cortex and in sensory association fields, as well as in medium-sized projection neurons of the striatum (extrapyramidal symptoms). During the fourth and final stage, the cortical pathology progresses into the temporal 


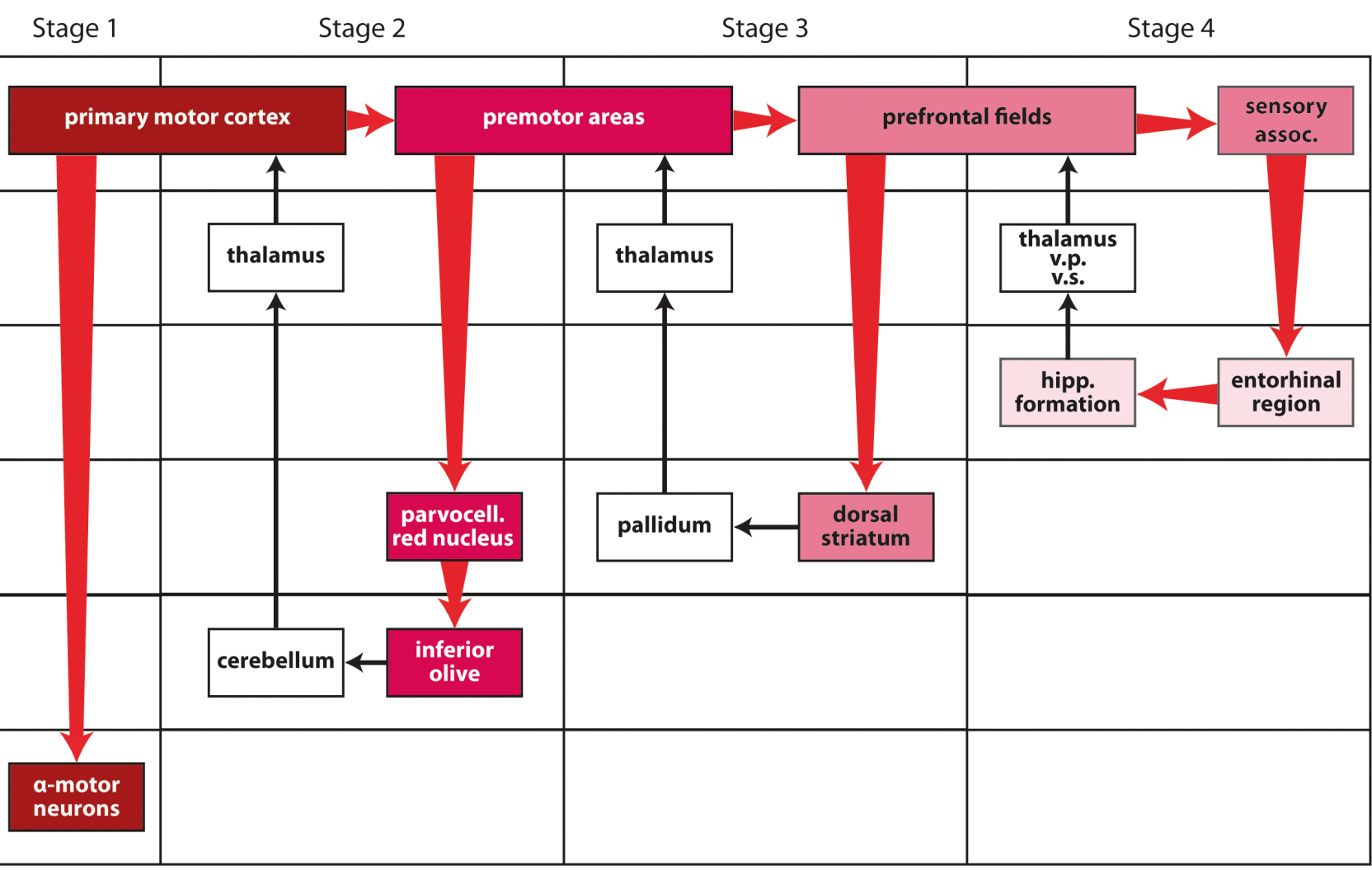

Figure 2 Schematic diagram showing the proposed development of TDP-43 lesions in structures that are under the direct control of corticofugal projections during four neuropathological stages of sporadic amyotrophic lateral sclerosis. Colour coding (stage 1, brown; stage 2, deep red; stage 3, light red; stage 4, pink) indicates the progression of the pathology during four successive neuropathological stages (see references 6 and 73). Uninvolved regions appear in white. The vertical red arrows indicate corticofugal pathways.

lobe and reaches the allocortical entorhinal region as well as the dentate fascia and Ammon's horn of the hippocampal formation (cognitive dysfunction) (figure 2). ${ }^{48}$

Remarkably, TDP-43 lesions develop only in structures that are under the direct control of corticofugal projections (figure 2). ${ }^{10}$ This provides support for the idea that the driving force behind the origins of the TDP-43 pathology must reside in the cerebral cortex itself, beginning in the neocortical motor fields, mediated via corticofugal axons and possibly the anterograde transport of toxic TDP-43 molecules. ${ }^{10}$ A site of origin outside of the cerebral cortex, in particular the spinal cord, is not compatible with this concept because it fails to explain the involvement of projection neurons in the parvocellular portion of the red nucleus or in the striatum, neither of which have connections with the spinal cord.

In sum, it is the neurons with corticofugal projections, that is, those giving rise to direct, monosynaptic projections to their subcortical targets, that develop TDP-43 pathology (figure 2), while neurons with predominantly corticopetal connectivities, the group of so-called non-thalamic nuclei with diffuse projections to the cerebral cortex (eg, the locus coeruleus, upper raphe nuclei and the magnocellular nuclei of the basal forebrain), are virtually spared. ${ }^{5} 10$

CM cells represent a distinct component of the corticobulbar/ corticospinal output. This component probably includes the Betz cells (corticobulbar/corticospinal neurons with largest cell bodies and fast-conducting axons) but also smaller projection neurons. ${ }^{54}$ In macaques it has been shown that the CM output comes mainly from the 'new M1' located in the most caudal portion of the primary motor cortex close to the central sulcus. ${ }^{21}$ Direct control of movement by the CST via its monosynaptic contacts is an increasingly important feature of primate motor systems, in contrast to the indirect cortical influence exerted on $\alpha$-motoneurons via interneurons that is found in non-primates. ${ }^{14}$

Corticospinal neurons in primates exhibit a number of key features which distinguish them from those in rodents, including short duration spikes, ${ }^{55}$ some fibres with fast conduction velocities ${ }^{54}$ and widespread expression of the fast $\mathrm{K}+$ channel Kv3.1b. ${ }^{56}$ These primate-specific features may in some way be related to the particular vulnerability of these neurons to TDP-43 pathology in humans.

It remains a possibility that the spread of the pathology to interconnected neurons takes place as a 'prion-like' cell-tocell transmission by means of the phylogenetically 'new' or recently evolved monosynaptic contacts. ${ }^{52}$ 57-59 Whether this might hold true for both CBT/CST projections but also for corticorubral and corticostriatal connectivities remains to be determined. However, a prion-like spread for TDP-43 protein associated with ALS has not yet been documented conclusively. ${ }^{60}$

Separately, in a case of Mills syndrome, a rare ALS phenotype characterised by a hemiparetic weakness, the hemisphere contralateral to the weakness, showed striking atrophy involving the primary motor cortex and the frontal and particularly the left temporal lobe. ${ }^{61}$ This was accompanied by neuronal loss, gliosis and TDP-43-positive neuronal and glial cytoplasmic inclusions. CD68 staining for microglia, a sensitive marker of axonal loss, showed axonal loss in the main crossed lateral CST but also the anterior CST carrying uncrossed descending fibres from the cortex to the cervical and thoracic spinal cord. Thus, involvement of the anterior CST, although not yet systematically studied in ALS, cannot be explained on a dying-back neurodegeneration, supports the wider concept of a cortical dying-forward mechanism. 


\section{Support from neuroimaging}

Even though there is now an extensive literature on neuroimaging in ALS, clearly showing a variety of abnormalities in many components of the brain, it is still not possible to directly determine a cortical origin of ALS from imaging studies. Current tractography methods do not allow unequivocal identification of CBT/CSTs because these fibres are heavily intermingled with other descending fibre systems, which far outnumber them. ${ }^{62}$ Nevertheless, inferences can be made from imaging in ALS, which point to the primacy of the cerebral cortex.

For example, structural and functional MRI, positron emission tomography (PET), and single photon emission-computed tomography (SPECT) studies all confirm frontotemporal impairment in ALS in approximately 50\% of patients, far more than those with overt cognitive dysfunction. Also diffusion tensor imaging (DTI) findings of reduced white matter integrity in the frontal, temporal and parietal lobes and, often early in the corpus callosum, suggest widespread white matter involvement may underlie both cognitive and other functional changes in ALS. ${ }^{63}$ These changes are independent of spinal cord pathology.

In addition, resting-state functional MRI (RS-fMRI), a technique that evaluates the spontaneous fluctuations in the blood oxygen level-dependent signals with subjects at rest, has documented focal degeneration of cortical motor areas is accompanied by a widespread effect on connected brain networks. ${ }^{64}$ This confirms that ALS is a multisystem disease affecting not only primary motor connections but also the connectivity between primary motor regions and supplementary motor and premotor regions.

Brain cortical thickness analysis in ALS demonstrates cortical thinning (of about 12\%) of the precentral gyrus and is most prominent in upper portions of the primary motor cortex which contribute to control muscles of the trunk and extremities, in keeping with limb-onset clinical presentation in the majority of ALS cases. ${ }^{65} 66$ The most disease-sensitive MRI patterns are located in cortical motor regions, and especially along tracts containing corticobulbar/corticospinal fibres (given the limitations mentioned above). Other parts of the frontal lobe, the temporal lobe, including the hippocampal formation, the parietal lobe and the insula, are commonly involved, whereas the occipital lobe is less frequently involved. ${ }^{67}$ Longitudinal analysis of a small numbers of patients with ALS has shown a decrease in cortical thickness in motor, temporal and frontoparietal cortices, as well as DTI changes in the CBT/CSTs tracts corpus callosum and frontal white matter. ${ }^{68}$ DTI studies, with tractography or connectome analysis, provide in vivo evidence for progressive expansion of white matter damage from motor to extramotor networks. ${ }^{6869}$

Neuroradiological techniques, using DTI and fractional anisotropy as well as RS-fMRI, have also been used for in vivo staging of ALS and shown to correlate with neuropathological TDP-43 data. ${ }^{40}$ Structural and functional connectivity is more impaired in regions topologically closer to the motor cortex relative to distant ones and structural connectivity is progressively impaired in ALS. ${ }^{70}$

PET has demonstrated variable cortical and subcortical abnormalities in ALS and ALS-FTD both with and in the absence of identified ALS genes. ${ }^{71}$ However, the potential of PET in ALS will require development of ligand markers directed to specific pathogenic hypotheses, for example, glutamate receptors, specific neuroinflammatory or protein markers, for example, TDP-43. SPECT has proved a practical and significant approach for recognising the clinical, pathological and genetic continuum between ALS and FTD. However, although PET has greater resolution than fMRI, it is limited by cost and availability. PET studies, using tracers sensitive to blood flow and metabolism (eg, radiolabelled water and flurodeoxyglucose), demonstrated consistent motor and extramotor cerebral pathology in ALS. Subsequently, 'ligand' PET used to identify specific cerebral neuronal receptor changes in ALS has provided evidence for a loss of cortical inhibition, widespread microglial activation and a striking reduction in serotonin-1A receptor binding. ${ }^{71}$

\section{FURTHER CONSIDERATIONS}

We have underscored the importance and uniqueness of the primate and in particular the human CM system to explain many early features of ALS, a singularly human disorder. Although the hallmark TDP-43 pathology, which is largely restricted to neurons that receive corticofugal projections, is recognised in animal (rodent) models of ALS, there have been, to date, very few in vivo animal studies exploring cortical aspects of ALS. ${ }^{72}$ Rodent models do not share fundamental aspects of descending motor pathways of primates, ${ }^{1456}$ nor do rodents have the critical cortical connectome, especially as it applies to the neocortex, disruption of which is so fundamental in ALS, as well as in Parkinson's and Alzheimer's diseases. ${ }^{5} 73$ Furthermore, rodent models do not replicate the interaction that ageing plays in human neurodegenerations. ${ }^{7475}$

Our view regarding the primacy of the human neocortex as driving ALS, if correct, clearly limits the extent to which rodent models of ALS can ultimately explain the complete pathogenicity of this disease, and greater benefit will be derived from efforts directed to the study of primates and in particular the human brain, as the initial site of ALS neurodegeneration.

However, because ALS is phenotypically quite variable, presenting in many different ways, at different ages and with variable progression, it is important to question if the cortical hypothesis of ALS as presented here is equally relevant to all forms of ALS. Do all ALS phenotypes reflect a continuum of one disease, or are they truly differing conditions? Our present position is that they are a continuum, but this question will only be answerable once the complete ALS genome is unravelled. A further consideration for the cortical hypothesis relates to neuronal and non-neuronal elements. We have stressed the importance of the CM system in this review, but clearly inhibitory neurons, interneurons and glia are of considerable importance in the overall pathogenesis of ALS. But none of this per se negates the primacy of the cortex in ALS.

There is substantial support for a long presymptomatic period in ALS, ${ }^{76}$ and one might reasonably speculate that its origin even lies in the developing neonatal brain, as is being established for a number of apparently adult onset neurological disorders. Elucidating unique features of human neocortical development and function may then help yield new insights into the aetiology of ALS. The protracted duration of both fetal development and the subsequent neurogenic period in humans, together with altered cell cycle dynamics, is posited to explain human-specific differences in neuron number and overall size of the neocortex. ${ }^{12} 77$ Separately, diverse genomic changes implicated in brain evolution include species-specific alterations to coding sequences, regulatory DNA, non-coding RNAs and gene gains or losses. The major challenge will be our ability to assess function of these differences in humans versus non-primates.

Correction notice Since this review was first published online the author name $\mathrm{K}$ Del Tredici has been updated. 
Acknowledgements The authors (HB, KDT) thank Mr David Ewert for technical assistance with the graphics (figure 2).

Contributors All authors contributed equally to this review paper.

Competing interests None declared.

Provenance and peer review Commissioned; externally peer reviewed.

(c) Article author(s) (or their employer(s) unless otherwise stated in the text of the article) 2017. All rights reserved. No commercial use is permitted unless otherwise expressly granted.

\section{REFERENCES}

1 Al-Chalabi A, Jones A, Troakes C, et al. The genetics and neuropathology of amyotrophic lateral sclerosis. Acta Neuropathol 2012;124:339-52.

2 Mackenzie IR, Neumann M, Bigio EH, et al. Nomenclature for neuropathologic subtypes of frontotemporal lobar degeneration: consensus recommendations. Acta Neuropathol 2009;117:15-18

3 Buratti E, Romano M, Baralle FE. TDP-43 high throughput screening analyses in neurodegeneration: advantages and pitfalls. Mol Cell Neurosci 2013;56:465-74.

4 Fallini C, Bassell GJ, Rossoll W. The ALS disease protein TDP-43 is actively transported in motor neuron axons and regulates axon outgrowth. Hum Mol Genet 2012;21:3703-18

5 Braak H, Del Tredici K. Neuroanatomy and pathology of Sporadic Parkinson's disease. Adv Anat Embryol Cell Biol 2009;201:1-119.

6 Charcot J. Sclerose laterale amytrophique. Oeuvres Compltes. Bureaux du Proges Medical 1874;2:249-66.

7 Kiernan JA, Hudson AJ. Changes in sizes of cortical and lower motor neurons in amyotrophic lateral sclerosis. Brain 1991;114 (Pt 2):843-53.

8 Eisen A, Calne D. Amyotrophic lateral sclerosis, Parkinson's disease and Alzheimer's disease: phylogenetic disorders of the human neocortex sharing many characteristics. Can J Neurol Sci 1992;19:117-23.

9 Eisen A, Weber M. The motor cortex and amyotrophic lateral sclerosis. Muscle Nerve 2001;24:564-73.

10 Braak H, Brettschneider J, Ludolph AC, et al. Amyotrophic lateral sclerosis - a model of corticofugal axonal spread. Nat Rev Neurol 2013;9:708-14.

11 Kiernan MC, Vucic S, Cheah BC, et al. Amyotrophic lateral sclerosis. Lancet 2011;377:942-55

12 Welniarz Q, Dusart I, Roze E. The corticospinal tract: Evolution, development, and human disorders. Dev Neurobiol 2016.

13 Isa T, Kinoshita M, Nishimura Y. Role of Direct vs. Indirect Pathways from the Motor Cortex to Spinal Motoneurons in the Control of Hand Dexterity. Front Neurol 2013;4:191.

14 Lemon RN. Descending pathways in motor control. Annu Rev Neurosci 2008:31:195-218.

15 Lemon RN. What drives corticospinal output? F1000 Biol Rep 2010;2:51.

16 Muir RB, Lemon RN. Corticospinal neurons with a special role in precision grip. Brain Res 1983:261:312-6.

17 Eisen A, Turner MR, Lemon R. Tools and talk: an evolutionary perspective on the functional deficits associated with amyotrophic lateral sclerosis. Muscle Nerve 2014;49:469-77.

18 Kuypers H. Anatomy of the descending pathways. In: Brookhart J, Mountcastle VB, eds. Handbook of Physiology - The Nervous System II American Physiological Society. Bethesda, MD, 1981:597-666.

19 Kuypers HG. Corticobular connexions to the pons and lower brain-stem in man: an anatomical study. Brain 1958:81:364-88.

20 Imatsubo T, Kuzuhara S, Kanemitsu A, et al. Corticofugal projections to the motor nuclei of the brainstem and spinal cord in humans. Neurology 1990;40:309-12

21 Rathelot JA, Strick PL. Subdivisions of primary motor cortex based on corticomotoneuronal cells. Proc Natl Acad Sci U S A 2009;106:918-23.

22 de Noordhout AM, Rapisarda G, Bogacz D, et al. Corticomotoneuronal synaptic connections in normal man: an electrophysiological study. Brain 1999:122 7:1327-40.

23 Barthélemy D, Grey MJ, Nielsen JB, et al. Involvement of the corticospinal tract in the control of human gait. Prog Brain Res 2011;192:181-97.

24 Fornia L, Ferpozzi V, Montagna M, et al. Functional Characterization of the Left Ventrolateral Premotor Cortex in Humans: A Direct Electrophysiological Approach. Cereb Cortex 2016

25 Eisen A, Kuwabara S. The split hand syndrome in amyotrophic lateral sclerosis. $J$ Neurol Neurosurg Psychiatry 2012;83:399-403.

26 Simon NG, Lee M, Bae JS, et al. Dissociated lower limb muscle involvement in amyotrophic lateral sclerosis. J Neurol 2015;262:1424-32.

27 Eisen A. Amyotrophic lateral sclerosis: a 40-year personal perspective. J Clin Neurosci 2009:16:505-12.

28 Petersen NC, Taylor JL, Murray NP, et al. Differential effects of low-intensity motor cortical stimulation on the inspiratory activity in scalene muscles during voluntary and involuntary breathing. Respir Physiol Neurobiol 2011;175:265-71.
29 Espadaler J, Rogić M, Deletis V, et al. Representation of cricothyroid muscles at the primary motor cortex (M1) in healthy subjects, mapped by navigated transcranial magnetic stimulation (nTMS). Clin Neurophysiol 2012;123:2205-11.

30 Simonyan K. The laryngeal motor cortex: its organization and connectivity. Curr Opin Neurobiol 2014;28:15-21.

31 Andersen PM. Mutation in C9orf72 changes the boundaries of ALS and FTD. Lancet Neurol 2012;11:205-7.

32 Phukan J, Pender NP, Hardiman O. Cognitive impairment in amyotrophic lateral sclerosis. Lancet Neurol 2007;6:994-1003.

33 Burrell JR, Halliday GM, Kril JJ, et al. The frontotemporal dementia-motor neuron disease continuum. Lancet 2016:388:919-31.

34 Eisen A, Lemon R, Kiernan MC, et al. Does dysfunction of the mirror neuron system contribute to symptoms in amyotrophic lateral sclerosis? Clin Neurophysiol 2015; 126:1288-94

35 Jelsone-Swain L, Persad C, Burkard D, et al. Action processing and mirror neuron function in patients with amyotrophic lateral sclerosis: an fMRI study. PLoS One 2015:10:e0119862

36 Eisen A, Vucic S. Fasciculation potentials: a diagnostic biomarker of early ALS? J Neurol Neurosurg Psychiatry 2013:84:948

37 de Carvalho M, Swash M. Physiology of the fasciculation potentials in amyotrophic lateral sclerosis: which motor units fasciculate? J Physiol Sci 2016

38 Gorges M, Müller HP, Lulé D, et al. Eye Movement Deficits Are Consistent with a Staging Model of pTDP-43 Pathology in Amyotrophic Lateral Sclerosis. PLoS One 2015:10:e0142546.

39 Schall JD. Neuronal activity related to visually guided saccadic eye movements in the supplementary motor area of rhesus monkeys. J Neurophysiol 1991;66:530-58.

40 Brettschneider J, Del Tredici K, Toledo JB, et al. Stages of pTDP-43 pathology in amyotrophic lateral sclerosis. Ann Neurol 2013:74:20-38.

41 Vucic S, Ziemann U, Eisen A, et al. Transcranial magnetic stimulation and amyotrophic lateral sclerosis: pathophysiological insights. J Neurol Neurosurg Psychiatry 2013;84:1161-70

42 Meyer BU, Röricht S, Gräfin von Einsiedel H, et al. Inhibitory and excitatory interhemispheric transfers between motor cortical areas in normal humans and patients with abnormalities of the corpus callosum. Brain 1995;118(Pt 2):429-40.

43 Menon P, Kiernan MC, Vucic S. Cortical hyperexcitability precedes lower motor neuron dysfunction in ALS. Clin Neurophysiol 2015;126:803-9.

44 Vucic S, Nicholson GA, Kiernan MC. Cortical hyperexcitability may precede the onset of familial amyotrophic lateral sclerosis. Brain 2008;131:1540-50.

45 Vucic S, Lin CS, Cheah BC, et al. Riluzole exerts central and peripheral modulating effects in amyotrophic lateral sclerosis. Brain 2013;136:1361-70.

46 Vucic S, Nicholson GA, Kiernan MC. Cortical excitability in hereditary motor neuronopathy with pyramidal signs: comparison with ALS. J Neurol Neurosurg Psychiatry 2010;81:97-100.

47 Wittstock M, Wolters A, Benecke R. Transcallosal inhibition in amyotrophic lateral sclerosis. Clin Neurophysiol 2007;118:301-7.

48 Burrell JR, Kiernan MC, Vucic S, et al. Motor neuron dysfunction in frontotempora dementia. Brain 2011;134:2582-94.

49 Eisen A, Kim S, Pant B, et al. ALS): a phylogenetic disease of the corticomotoneuron? Muscle Nerve 1992;15:219-24.

50 van Es MA, Van Vught PW, Blauw HM, et al. ITPR2 as a susceptibility gene in sporadic amyotrophic lateral sclerosis: a genome-wide association study. Lancet Neurol 2007:6:869-77.

51 Kwak S, Kawahara Y. Deficient RNA editing of GluR2 and neuronal death in amyotropic lateral sclerosis. J Mol Med 2005;83:110-20.

52 Braak H, Ludolph AC, Neumann M, et al. Pathological TDP-43 changes in Betz cells differ from those in bulbar and spinal $\alpha$-motoneurons in sporadic amyotrophic lateral sclerosis. Acta Neuropathol 2017:133:79-90.

53 Büttner-Ennever JA. The extraocular motor nuclei: organization and functional neuroanatomy. Prog Brain Res 2006:151:95-125.

54 Firmin L, Field P, Maier MA, et al. Axon diameters and conduction velocities in the macaque pyramidal tract. J Neurophysio/ 2014:112:1229-40.

55 Vigneswaran G, Kraskov A, Lemon RN. Large identified pyramidal cells in macaque motor and premotor cortex exhibit "thin spikes": implications for cell type classification. J Neurosci 2011:31:14235-42.

56 Soares D, Goldrick I, Lemon RN, et al. Expression of Kv3.1b potassium channel is widespread in macaque motor cortex pyramidal cells: A histological comparison between rat and macaque. J Comp Neurol. In Press. 2017;525:2164-74.

57 Smethurst P, Newcombe J, Troakes C, et al. In vitro prion-like behaviour of TDP-43 in ALS. Neurobiol Dis 2016;96:236-47.

58 Guo L, Shorter J. Biology and Pathobiology of TDP-43 and Emergent Therapeutic Strategies. Cold Spring Harb Perspect Med 2016:a024554.

59 Feiler MS, Strobel B, Freischmidt A, et al. TDP-43 is intercellularly transmitted across axon terminals. J Cell Biol 2015:211:897-911.

60 Stopschinski BE, Diamond MI. The prion model for progression and diversity of neurodegenerative diseases. Lancet Neurol 2017;16:323-32.

61 Bäumer D, Butterworth R, Menke RA, et al. Progressive hemiparesis (Mills syndrome) with aphasia in amyotrophic lateral sclerosis. Neurology 2014;82:457-8 
62 Tomasch J. The numerical capacity of the human cortico-pontocerebellar system. Brain Res 1969;13:476-84.

63 Menke RA, Agosta F, Grosskreutz J, et al. Neuroimaging endpoints in Amyotrophic Lateral Sclerosis. Neurotherapeutics 2017;14:11-23.

64 Schulthess I, Gorges M, Müller HP, et al. Functional connectivity changes resemble patterns of pTDP-43 pathology in amyotrophic lateral sclerosis. Sci Rep 2016;6:38391

65 Turner MR, Agosta F, Bede P, et al. Neuroimaging in amyotrophic lateral sclerosis. Biomark Med 2012;6:319-37.

66 Agosta F, Valsasina P, Riva N, et al. The cortical signature of amyotrophic lateral sclerosis. PloS One 2012;7:e42816.

67 Chiò A, Pagani M, Agosta F, et al. Neuroimaging in amyotrophic lateral sclerosis: insights into structural and functional changes. Lancet Neurol 2014;13:1228-40.

68 Kassubek J, Müller HP, Del Tredici K, et al. Diffusion tensor imaging analysis of sequential spreading of disease in amyotrophic lateral sclerosis confirms patterns of TDP-43 pathology. Brain 2014;137:1733-40.

69 Verstraete $\mathrm{E}$, Veldink JH, van den Berg LH, et al. Structural brain network imaging shows expanding disconnection of the motor system in amyotrophic lateral sclerosis. Hum Brain Mapp 2014;35:1351-61.
70 Schmidt R, Verstraete E, de Reus MA, et al. Correlation between structural and functional connectivity impairment in amyotrophic lateral sclerosis. Hum Brain Mapp 2014;35:4386-95.

71 Turner MR, Bowser R, Bruijn L, et al. Mechanisms, models and biomarkers in amyotrophic lateral sclerosis. Amyotroph Lateral Scler Frontotemporal Degener 2013;14:19-32.

72 Philips T, Rothstein JD. Rodent models of Amyotrophic Lateral Sclerosis. Curr Protoc Pharmacol 2015:695:1-21.

73 Owen JP, Li YO, Ziv E, et al. The structural connectome of the human brain in agenesis of the corpus callosum. Neuroimage 2013;70:340-55

74 Cookson MR. Evolution of neurodegeneration. Curr Biol 2012;22:R753-R761.

75 Uchida A, Sasaguri H, Kimura N, et al. Non-human primate model of amyotrophic lateral sclerosis with cytoplasmic mislocalization of TDP-43. Brain 2012;135:833-46.

76 Eisen A, Kiernan M, Mitsumoto $\mathrm{H}$, et al. Amyotrophic lateral sclerosis: a long preclinical period? J Neurol Neurosurg Psychiatry 2014;85:1232-8.

77 Biane JS, Scanziani M, Tuszynski MH, et al. Motor cortex maturation is associated with reductions in recurrent connectivity among functional subpopulations and increases in intrinsic excitability. J Neurosci 2015:35:4719-28. 